\title{
The biopsychosocial condition of childhood cancer survivors in the transition towards adult care: a national survey from the joint pediatric and adult transition care group
}

\author{
${ }^{1}$ Paediatric Onco-Haematology, Stem Cell Transplantation and Cellular Therapy Division, City of Science and Health of Turin, \\ Regina Margherita Children's Hospital, Turin, Italy, E-mail: giulia.zucchetti1@gmail.com \\ ${ }^{2}$ Transition Unit for Childhood Cancer Survivors, City of Science and Health of Turin, Turin, Italy
}

\begin{abstract}
:
Background: To provide successful transfer from childhood to adult-oriented healthcare is one of the priorities of survivorship care plans.

Purpose: This study describes adolescent and young adult childhood cancer survivors' conditions at the moment of the transition to adult care deepening their biological, psychological, social and assistant state and their associations with socio-demographic and clinical characteristics.

Methods: A biopsychosocial check-list in four health domains (biological, psychological, social and assistant) was filled in by healthcare professionals (oncologists, psychologists, social workers and nurses) through qualitative interviews and clinical observations of 79 survivors ( $58 \%$ boys; $\mathrm{M}_{\text {age }}=20$ years old) at the moment of the transition from the Pediatric Oncology Unit to the Transition Unit of the Childhood Cancer Survivors.

Results: At the moment of transition, 38\% of survivors showed a positive condition in all the four health domains without any kind of impairment. Biological (37\%) and psychological areas (44\%) were found to be those with major incidence of impairments. Association phenomena were found between psychological and social condition $(p<0.05)$ and between social and assistant condition $(p<0.05)$. Biological condition was also significantly associated with the type of cancer $(\chi=6,2414, p<0.05)$.

Conclusion: Although many survivors entered in adult care system without any impairment, the biopsychosocial approach highlighted that there is a presence of impairments in at least one of the main health domains. Keywords: biopsychosocial, childhood cancer survivors, psycho-oncology, transition
\end{abstract} DOI: 10.1515/ijamh-2018-0189

Received: August 18, 2018; Accepted: October 4, 2018

\section{Introduction}

Pediatric oncology is seen as a major success in current medicine. New drugs, precision medicine, treatment reduction and holistic care are some of the factors responsible for the drastic decrease of the childhood cancer mortality rate, thus increasing the number of survivors [1]. Currently, in Europe there are approximately 400,000 long term survivors of which 30,000 are in Italy. The presence of so many childhood cancer survivors has created new central clinical issues in terms of late effects' management and quality of life preservation against long -erm morbidities of therapy. Unfortunately, over $40 \%$ of childhood cancer survivors develope severe or chronic health conditions such as second malignancies, cardiac and vascular abnormalities, pulmonary complications. Moreover, these effects are always associated with stress, isolation and depression state [2] and body image discomfort [3]. For these reasons, the need for a structured long-term follow-up (LTFU) system, aimed at countering potential health risks and supporting beneficial behaviors among youth survivors, has been widely recognized.

The involvement in a formalized follow-up, in addition to monitoring late effects, promote and facilitate an important and challenging process for the patient: the transition from a child-oriented to adult-oriented healthcare system. This is a really significant moment, especially for youths who have special healthcare needs, and 
this is often complicated by another important moment that these youths have to deal with: the transition from adolescence to young adulthood. These turning points can affect each other, thus justifying the need to consider the transition of care as one of the priorities of national healthcare systems [4], [5]. The literature shows that many adolescent and young adult (AYA) cancer survivors did not feel adequately prepared to transition to independent adult care, they perceived themselves to have low self-esteem and low cognitive and interpersonal abilities [6] and they felt themselves emotionally dependent on their family and/or pediatric providers [7]. Nathan and colleagues [8] underlined as AYA cancer survivors face a unique range of biological and psychosocial issues that may affect their transition such as physical health and function, fertility preservation, emotional discomfort and risk behaviors. A study about the criteria of the transition process confirmed these issues revealing the very complex limitations for success in transition in two main fields of concerns: medical and mental health impairments, such as severe physical and biological late effects, and social and educational barriers, such as educational achievement and lower income [5]. For overcoming these obstacles, specific educational and psychological programs should be proposed at the time of treatment, in order to prepare youths for the transition experience. The critical condition of AYA cancer survivors is also confirmed by healthcare professionals, especially pediatricians [9] who often continue to care about survivors for over 25 years. These pediatricians are more likely to perceive survivors psychosocial needs as barriers to transfer, such as attachment to provider and unstable social situations.

Confirming the critical importance of the AYA cancer survivors transition experience for their future general health status, the European PanCareSurFup Consortium has provided a first formal definition of transition. Transition of childhood cancer survivors was defined as an active, multidisciplinary process to enable survivors to effectively transfer from child-centered to adult-oriented healthcare systems. The transition process should be flexible and consider the medical, psychosocial, educational and vocational needs of survivors and promote a healthy lifestyle and management [10]. Therefore, there is a transnational agreement about the relevance of the AYA cancer survivors' transition and, for this reason, a global exploration about the numerous needs of survivors (in terms of biological, psychological, social and assistant needs) at the moment of the transition seems to be strictly necessary and urgent. This objective is particularly important because it could allow professionals to design formal transition programs and specific guidelines taking into account all these issues in order to sustain the AYA cancer survivors' engagement with the new health services.

However, to the best of our knowledge, clinical research has never investigated the condition of survivors in the specific moment of transition towards the adult care system. Moreover, no study has examined the mentioned needs among AYA cancer survivors at the moment of the transition to the adult system. This study intends to cover this lack by exploring the condition of the AYA adopting a biopsychosocial perspective. While the biopsychosocial model has gained prominence over the years, it has not been studied much in AYA cancer survivors approaching transitional care. The possibility to obtain a global vision about the health state of the AYA cancer survivors at the moment of the transition could permit pediatric and adult professionals to share possible facilitators of the process improving the AYA engagement in LTFU in adult care health system.

The first aim of this study was to highlight the most common biopsychosocial survivors' profiles and to describe the rate of impairments in the four health domains, biological, psychological, social and assistant. The second aim was to explore possible association phenomena among the four health domains and the possible association between the four health domains and socio-demographic (i.e. gender, age at diagnosis, age at the transition) and clinical characteristic (type of diagnosis).

\section{Methods}

\section{Setting and participants}

Participants were recruited from the Paediatric Onco-Haematology Division at the moment of transition to the Transition Unit for Childhood Cancer Survivors of the City of Health and Science of Turin from January 2015 to December 2017. This Transition Unit is unique in Italy and it is a specialized structure exclusively dedicated to the LTFU of cancer survivors. This unit is headed by an internist/endocrinologist and organized with a network of multi-disciplinary and dedicated specialists. They collaborate with the pediatric oncologists of the Pediatric Hematology-Oncology Unit in order to better prepare survivors, families and professionals through a formal transitional care program. This program consists in a clinical joint visit with pediatrics and adult professionals, which provide educational suggestions and a structured interview with psychologists dedicated to the AYA cancer survivors during which their quality of life is investigated through a biopsychosocial approach. This program is formalized by the hospital's units so the ethical approval of the Research Ethical Committee was not necessary. However every participants having provided written informed consent to participate to the for- 
mal transition program meant the consent was kept in their hospital clinical records. Long-term survivors are usually transitioned when they are aged over 18 years and they are off-therapy for at least 5 years.

Eligible criteria were: (i) age at diagnosis under 18 years; (ii) at least 5 years of survival after the first cancer diagnosis; and (iii) at least one visit after the 18th birthday. Each year approximately 35 survivors transit to adult care.

\section{Procedure}

At the moment of the transition from pediatric to adult care for each AYA care survivors, four professionals representing specific health areas, pediatric oncologist, psychologist, social worker and nurse, compiled a biopsychosocial check-list, whose indicators reflect the major barriers to transition highlighted by the literature (e.g. the presence of late effects; psychological discomfort; vocational planning absence; low self-healthcare management) identifying biological, psychological, social and assistance impairments objectively detectable through observation and interview by clinicians. The biological area covers different aspects about medical and clinical history, such as fertility preservation, eating disorders, important effects of chemotherapy, radiotherapy or surgery, relapses, second tumors and bodily changes. The psychological area considers psychological and cognitive impairments, lifestyle risks and feelings such as anxiety and depression. The social area covers several social risk situations such as lack of employment or school abandonment and critical economic and housing situations. Finally, the assistant area covers several difficulties in taking care of yourself. For each area (biological, psychological, social and assistant) the dedicated clinicians attributed a score about the presence or absence $(1 / 0)$ of impairments. Every survivor was categorized with an absence or a presence of impairment for each of the four health domains investigated. Data about age at diagnosis, age at transition, type of diagnosis and gender were also collected.

\section{Data analysis}

First, a descriptive analysis was performed on the four health domains in order to identify the most common biopsychosocial profile of survivors and to calculate the incidence of impairments for each domain.

Secondly, the phi coefficient $(\phi)$ and chi-squared $\left(\chi^{2}\right)$ test were performed to measure, respectively, possible associations among health domains and between health domains and socio-demo/clinical variables.

The $\phi$ coefficient is a measure of association for two binary variables and its functioning is strictly similar to the Pearson correlation coefficient. The $\chi^{2}$ test is a measure of the association for categorical variables based on the comparison between observed and expected frequencies in a contingency table.

\section{Results}

From January 2015 to December 2017 the total number of AYA survivors scheduled for the transition were 103. Ninety-four AYA cancer survivors transferred to the adult system while nine of them have not transferred for different reasons (the majority of these survivors did not show up for clinical appointments). Of the 94 AYA cancer survivors, for 15 of them it was impossible to complete the entire check-list for all the four domains, so these 15 AYA survivors were excluded from the research.

The final sample of the research was composed of 79 AYA cancer survivors at the moment of the transition of care to the adult system ( 46 boys, $58 \% ; 33$ girls $42 \%$ ). The average age at the time of the study was 20 years (range 18-25) and the average age at diagnosis was 8.5 (range 1-17). Participants had a variety of diagnoses: the most common were lymphoblastic leukemia and other types of leukemia $(42,53 \%)$, lymphoma $(20,25 \%)$ and brain and bone tumors $(17,12 \%)$ (Table 1$)$.

Table 1: Descriptive analysis of the overall group.

\begin{tabular}{|c|c|c|c|}
\hline \multirow[t]{2}{*}{ Variable } & \multirow[t]{2}{*}{ Category } & \multicolumn{2}{|c|}{ Group } \\
\hline & & $\mathrm{n}$ & $\%$ \\
\hline \multirow[t]{2}{*}{ Gender } & Male & 46 & 58 \\
\hline & Female & 33 & 42 \\
\hline \multirow[t]{2}{*}{ Age at diagnosis } & $0-5$ & 18 & 23 \\
\hline & $6-13$ & 37 & 47 \\
\hline
\end{tabular}




\begin{tabular}{llrl} 
& $14-18$ & 24 & 30 \\
Age at transition & 18 & 18 & 23 \\
& Over 18 & 61 & 77 \\
Type of cancer & Leukemia & 42 & 53 \\
& Lynphomas & 20 & 25 \\
Biological condition & Brain and bones tumors & 17 & 12 \\
& Absence of impairments & 50 & 63 \\
Psychological condition & Presence of impairments & 29 & 37 \\
Social condition & Absence of impairments & 44 & 56 \\
& Presence of impairments & 35 & 44 \\
Assistance condition & Absence of impairments & 61 & 78 \\
& Presence of impairments & 18 & 22 \\
& Absence of impairments & 71 & 90 \\
\hline
\end{tabular}

Considering the survivor's profile as a combination of the condition on the overall four health domains, descriptive analysis indicated that the most common AYA cancer survivors' profile $(n=30,38 \%)$ is characterized by an absence of impairment in all the four health domains. Impairments in psychological and biological areas $(\mathrm{n}=10,13 \%)$ and a unique specific impairment in the biological area $(\mathrm{n}=10,13 \%)$ represented the two second most common AYA cancer survivors' profiles. Three survivors (4\%) showed impairments in all the four health domains.

Considering the individual incidence for each health domain, data showed an incidence of $34 \%(n=29)$ of biological impairment, $44 \%(n=35)$ of psychological impairment, $22 \%$ of social impairment $(n=18)$ and $10 \%$ of assistant impairment $(\mathrm{n}=8)$ (Table 1$)$.

In respect to associations among health domains, Table 2 summarizes the correlation matrix. Two significant associations were found: psychological condition and social condition were positively associated $(\phi=0.43, \mathrm{p}<$ $0.05)$ and social condition and assistance condition were also positively associated $(\phi=0.42, p<0.05)$. No other significant associations among the four health domains were highlighted.

Table 2: Correlation matrix of the health domains.

\begin{tabular}{lrrr}
\hline & $\mathbf{1}$ & $\mathbf{2}$ & $\mathbf{3}$ \\
\hline 1. Biological condition & - & & \\
2. Psychological condition & 0.22 & - & - \\
3. Social condition & 0.02 & $0.43^{\mathrm{a}}$ & $0.42^{\mathrm{a}}$ \\
4. Assistance condition & 0.18 & 0.29 & - \\
\hline
\end{tabular}

${ }^{a} p<0.05$.

The $\chi^{2}$ analysis between the four health domains and socio-demo/clinical variables showed that the biological condition was significantly associated with the type of diagnosis $\left(\chi^{2}=6,2414, p<0.01\right)$. The incidence of biological impairments was significantly higher for survivors who had brain and bone tumors. Results are summarized in Table 3. No other significant associations between health domains and socio-demo/clinical variables were found.

Table 3: Association between biological condition and type of cancer.

\begin{tabular}{|c|c|c|c|c|}
\hline & Leukemia & Lymphoma & Brain and bone tumor & Total \\
\hline \multicolumn{5}{|c|}{ Absence of biological impairments } \\
\hline $\mathrm{n}$ & 25 & 17 & 8 & 50 \\
\hline Row, \% & $50 \%$ & $34 \%$ & $16 \%$ & $100 \%$ \\
\hline Column, \% & $60 \%$ & $85 \%$ & $48 \%$ & $63 \%$ \\
\hline \multicolumn{5}{|c|}{ Presence of biological impairments } \\
\hline $\mathrm{n}$ & 17 & 3 & 9 & 29 \\
\hline Row, \% & $59 \%$ & $10 \%$ & $31 \%$ & $100 \%$ \\
\hline Column, \% & $40 \%$ & $15 \%$ & $52 \%$ & $37 \%$ \\
\hline \multicolumn{5}{|l|}{ Total } \\
\hline $\mathrm{n}$ & 42 & 20 & 17 & 79 \\
\hline Row, \% & $53 \%$ & $25 \%$ & $12 \%$ & $100 \%$ \\
\hline Column, \% & $100 \%$ & $100 \%$ & $100 \%$ & $100 \%$ \\
\hline
\end{tabular}


$\chi^{2}=6,2414, p<0.05$

\section{Conclusion}

Transition of care from the pediatric to the adult system is one of the most important turning points, especially for youths with special healthcare needs. For some AYA cancer survivors, the process of care transition presents particular challenges, because it also coincides with developmental transitions of emerging adulthood. The success of this transfer is mandatory because it allows the AYA cancer survivors to achieve the knowledge and to get the necessary skills to self-manage their follow-up care in adult healthcare systems, by limiting their sense of isolation and abandonment from the previous healthcare system.

As the theoretical framework showed, the barriers to the successful transition are related not only to medical and biological issues of survivors, but also to psychosocial and assistant ones. This condition of frailty in such a delicate moment of transition from the pediatric to the adult care system can have serious implications and consequences on survivors' conditions. However, to date, there still seems to be little knowledge about the conditions of survivors who are going to face the transition process. To the best of our knowledge a biopsychosocial perspective on the study about the condition of the AYA survivors at the moment of the transition has not already been applied.

Considering health impairments, many AYA cancer survivors do not present any impairment in biological, psychological, social and assistant domains. This result has been obtained thanks to the survivors' participation in several educational and psychosocial surveillance activities during their follow-up: these activities probably allowed them to face, in a timely fashion, any difficulties and to get prepared for the transition phase. Furthermore, the absence of biological impairments could reflect the improvement of the late effects of toxicity management thanks to the international long-term follow-up guidelines for the screening and management of late effects in survivors of childhood cancer that are followed and adopted by the clinical staff (survivorshipguidelines.org). These encouraging results reflect the increasing attention to quality of life for youth survivors that moves from "how long" people live after diagnosis to "how well" people can expect to live from diagnosis onward.

Despite this positive evidence, a critical subset of AYA survivors at the moment of the transition exists and should be highlighted. Although in a small percentage, several AYA cancer survivors present a common profile of biological impairments and a profile with both biological and psychological impairments. Moreover, the individual rate of impairments is higher for biological and psychological conditions than for social and assistant ones.

These results are consistent with other studies in which biological and psychological conditions have been shown as the two main barriers to transition.

The high rate of biological and psychological impairments in our group of survivors indicates that specific critical issue such as physical late effects, body concerns, sexual and reproductive issues and emotion and mental dysfunctions are not uncommon. These results are similar to those reported from other studies who have taken into account survivors' samples [11], but to date no result was available on the AYA cancer survivors' group at the moment of transition. The relevant incidence of biological and psychological impairments confirms the need of professionals (especially physicians and psychologists) dedicated to the AYA cancer survivors to be able to understand the unique medical and psychosocial needs of survivors and to be aware of the resources that can assist in navigating the transition phase. This approach is even more important for those AYA cancer survivors who have impairments in all domains, who need to be sustained in the transition process through a close multidisciplinary collaboration among pediatric and adult professionals.

Another important result of the study is the association between psychological and social impairments. Social domains, in terms of educational and professional attitudes and economic and family conditions, is highlighted by many studies as another major deficit in the transition process and as an obstacle faced by AYA cancer survivors [12]. During the interview conducted by social workers it emerged that many of the participants had lower educational and vocational attainment: they did not feel adequate to study and they have poor work-related outcomes. These social impairments undoubtedly have an impact on the AYA psychological state by enhancing isolation and feelings of suffering. Confirming this association, our study demonstrated that AYA cancer survivors with a positive psychological condition have also a satisfactory condition in different aspects of social life (work, vocation, family, etc.) and vice versa; for this reason, a synergic collaboration between psychologists and social workers, in order to empower psychological and social AYA cancer survivors' conditions, is already recommended during the various phases of cancer survivorship. The rationale is the same for the association between social and assistant conditions found in the study: survivors with a positive social adaptation are more cooperative and proactive in the care of their health status and vice versa. 
The previous associations suggest the opportunity for the generation of a virtuous circle in which psychological, social and assistant conditions influence each other improving the general well-being of survivors: the collaboration among professionals who individually deal with these domains is fundamental. Finally, an expected result was the strong relationship between biological impairment and type of diagnosis. Even at the time of the transition to the adult care system, it is important to take into account the different consequences of some types of childhood cancer such as brain and bone tumors in terms of long-term late effects. In line with the literature, this study showed that also at the moment of the transition towards the adult system AYA cancer survivors previously diagnosed with brain and bone tumors suffered for more biological impairments compared to their peers previously diagnosed with other types of tumors such as leukemia or lymphoma. Therefore, even more for brain and bone tumors survivors, a positive transition experience is required in order to allow them to have a strictly addressed long-term follow-up.

A potential limitation of this study was that the transition status was determined from the healthcare's point of view limiting a complete assessment reachable together with participants' self-reports. Also, its crosssectional design limits causal relationships being shown from observed variables.

Nonetheless, this study highlights specific unmet biopsychosocial and assistance associations: some of these were already reported among youths during the survivorship phase, but never explored at the moment of the transition.

A national joint model which incorporates both pediatric and adult clinicians is one of the best choices in providing an optimal transition of care of childhood cancer survivors, as that it is built around facilitating communication among all key stakeholders and emphasizing patient-friendly services that minimize patient stressors [13].

All potential healthcare providers must be aware of AYA cancer survivors' possible psychosocial late effects as for some professionals in the adult medical setting their identification could be challenging at the moment of the transition.

The presence and the persistence of clinically significant complications among AYA cancer survivors in place at the time of transition are important issues that make their management as one of the major goals of the oncology medical system.

Although psychosocial screening is considered a standard of care in long-term follow-up care for survivorship [14], this should also exist for the moment of the transition to adult care.

\section{Acknowledgments}

We are grateful to Dr. Tatiana Moda for language assistance and to Dr. Filippo Candela, PhD, for data analysis assistance.

\section{Compliance with ethical standards}

\section{Funding: None.}

\section{Conflict of interest statement: None.}

Ethical approval: All procedures performed in the study involving human subjects were in accordance with the ethical standards of the institutional research committee (Prot. $\mathrm{N}^{\circ} 0018330 \mathrm{~A} / 2.4 .8$ ). This article does not contain any studies with animals performed by any of the authors.

Informed consent: Informed consent was obtained from all individuals participants included in the study.

\section{References}

[1] Armstrong GT, Chen Y, Yasui Y, Leisenring W, Gibson TM, Mertens AC, et al. Reduction in late mortality among 5-year survivors of childhood cancer. N Engl ] Med. 2016;374(9):833-42.

[2] Mertens AC, Brand S, Ness KK, Li Z, Mitby PA, Riley A, et al. Health and well-being in adolescent survivors of early childhood cancer: a report from the Childhood Cancer Survivor Study. Psychoncology. 2014;23(3):266-75.

[3] Zucchetti C, Bellini S, Bertolotti M, Bona F, Biasin E, Bertorello N, et al. Body image discomfort of adolescent and young adult hematologic cancer survivors. J Adolesc Young Adult Oncol. 2017;6(2):377-80.

[4] Freyer DR. Transition of care for young adult survivors of childhood and adolescent cancer: rationale and approaches.] Clin Oncol. 2010;28(32):4810-8. 
[5] Frey E, van der Pal H. Transitional care of a childhood cancer survivor to adult services: facilitating the process of individual access to different models. Curr Opin Support Palliat Care. 2013;7(3):309-13.

[6] Patenaude AF, Kupst M]. Psychosocial functioning in pediatric cancer. ] Pediatr Psychol. 2005;30(1):9-27.

[7] Henderson TO, Friedman DL, Meadows AT. Childhood cancer survivors: transition to adult focused risk-based care. Pediatrics. 2010;126(1):129-36.

[8] Nathan PC, Hayes-Lattin B, Sisler ]]. Critical issues in transition and survivorship for adolescents and young adults with cancers. Cancer. 2011;117(S10):2335-41.

[9] Kenney LB, Melvin P, Fishman LN, O'Sullivan-Oliveira ], Sawicki CS, Ziniel S, et al. Transition and transfer of childhood cancer survivors to adult care: a national survey of pediatric oncologists. Pediatr Blood Cancer. 2017;64(2):346-52.

[10] Mulder RL, van der Pal H]H, Levitt CA, Skinner R, Kremer LCM, Brown MC, et al. Transition guidelines: an important step in the future care for childhood cancer survivors. A comprehensive definition as groundwork. Eur ] Cancer. 2016;54:64-8.

[11] Bitsko M], Cohen D, Dillon R, Harvey ], Krull K, Klosky JL. Psychosocial late effects in pediatric cancer. Survivors: a report from the children's oncology group. Ped Blood Cancer. 2016;63(2):337-43.

[12] Kirchhoff AC, Krull KR, Ness KK, Armstrong GT, Park ER, Stovall M, et al. Physical, mental, and neurocognitive status and employment outcomes in the childhood cancer survivor study cohort. Cancer Epidemiol Biomarkers Prev. 2011;20(9):1838-49.

[13] Sadak KT, Neglia JP, Freyer DR, Harwood E. Identifying metrics of success for transitional care practices in childhood cancer survivorship: a qualitative study of survivorship providers. Pediatr Blood Cancer. 2017;64(11). DOI: 10.1002/pbc. 26587.

[14] Lown EA, Phillips F, Schwartz LA, Rosenberg AR, Jones B. Psychosocial follow-up in survivorship as a standard of care in pediatric oncology. Pediatr Blood Cancer. 2017;62(S5):S514-84. 